\title{
Controversias con los medicamentos sintomáticos de acción lenta para la osteoartritis (SYSADOAs)
}

\section{Maritza Vidal Wilman', Luis Fernando Vidal Neira ${ }^{2 *}$}

1. Médico asistente del Centro de Diagnóstico de Osteoporosis y Enfermedades Reumáticas (CEDOR), Lima, Perú.

2. Médico jefe del servicio de Reumatología del Hospital María Auxiliadora. Director Médico del Centro de

Diagnóstico de Osteoporosis y Enfermedades Reumáticas (CEDOR), Lima, Perú.

International Osteoporosis Foundation, Delegado de Perú y Países Andinos.

*Correspondencia: Ifvidaln@hotmail.com

\section{Resumen}

La osteoartritis es una enfermedad de patogenia compleja y etiología multifactorial, clínicamente se caracteriza por su naturaleza heterogénea con expresiones fenotípicas distintas. El diagnóstico clásicamente se ha establecido en base a la combinación de las características clínicas y los hallazgos radiológicos compatibles con cambios degenerativos en la articulación afectada.

Entre los medicamentos disponibles para el manejo de la osteoartritis se cuenta con los analgésicos de acción rápida (Ej. Paracetamol, antiinflamatorios no esteroideos); los medicamentos sintomáticos de acción lenta (denominados SYSADOAs por sus siglas en inglés: Symptomatic Slow-Acting Drugs for Osteoarthritis); y finalmente se considera un tercer grupo, los medicamentos modificadores de la enfermedad (denominados también DMOA por sus siglas en inglés: "Disease modifying OA drugs"), pero como grupo es inexistente, porque ningún medicamento ha demostrado el potencial para remodelar o renovar la estructura de la articulación afectada. Se reconoce que algunos de los SYSADOA podrían tener también efecto estructural.

Se ha propuesto que un SYSADOA, debe de cumplir cuatro objetivos: Proporcionar alivio de los síntomas, disminuir el requerimiento de medicación sintomáti- ca concomitante (analgésicos, AINEs, etc.), prevenir el daño estructural y evitar (o retardar) el requerimiento de cirugía de reemplazo articular. Diferentes estudios clínicos y metanálisis encuentran evidencia que apoya la eficacia sobre el control del dolor de los SYSADOAs, pero con una heterogeneidad importante.

El propósito de este artículo es revisar las razones por las cuales existe discrepancia sobre la eficacia sintomática de los SYSADOAs, entre las cuales se encuentran la baja calidad metodológica de algunos estudios clínicos, las características y la naturaleza de la molécula específicamente empleada, las variaciones en la molécula original, el estatus de registro (medicamentos de venta libre o bajo prescripción), el procesamiento y la fuente de financiación de los estudios clínicos entre otros.

Palabras clave:

Osteoartritis; SYSADOAs. 


\section{Introducción}

La osteoartritis es una enfermedad de patogenia compleja y etiología multifactorial que traduce la falla de la articulación causada por diferentes factores y se asocia con cambios estructurales en el cartílago y en las demás estructuras que conforman la articulación. Clínicamente se caracteriza por su naturaleza heterogénea con expresiones fenotípicas distintas. La edad de inicio, el patrón de compromiso articular y la tasa de progresión son variables entre las personas afectadas y dependen también del lugar anatómico comprometido [1].

El diagnóstico de esta enfermedad clásicamente se ha establecido en base a la combinación de las características clínicas y los hallazgos radiológicos compatibles con cambios degenerativos en la articulación afectada. Sin embargo; según los últimos criterios para la osteoartritis de rodillas propuestos por la European League Against Rheumatism (EU$L A R)$, el diagnóstico se puede establecer sin la confirmación radiológica o de otros estudios de imagen, siempre que se cumplan los criterios clínicos [2].

Las medidas terapéuticas disponibles se pueden agrupar en cuatro categorías: manejo no farmacológico, manejo farmacológico, medicina alternativa o complementaria y cirugía. En general es recomendable iniciar el tratamiento utilizando las medidas más seguras y menos invasivas, para posteriormente progresar hacia terapias más agresivas si la situación clínica lo amerita [3].

Las intervenciones no farmacológicas forman una parte esencial en el manejo de los pacientes con osteoartritis y deben ser las primeras en recomendarse desde el inicio de la enfermedad y durante todo el curso de la misma; debiéndose adaptar de acuerdo a la naturaleza de los síntomas. Una parte importante es la educación al paciente y a la familia sobre la importancia de las medidas terapéuticas y la adherencia a éstas para obtener mejores resultados [4].

Las medidas no farmacológicas son de bajo costo, incorporan alternativas de automanejo que pueden ejecutarse en casa y tienen un impacto importante sobre el paciente [5]; además de proporcionar beneficios adicionales para la salud como bajar de peso, disminuir el riesgo cardiovascular, evitar el sedentarismo, prevenir la sarcopenia; y mejorar la autoestima y la seguridad.

Para el manejo farmacológico, existe una serie bastante amplia de medicamentos disponibles, pero en general se pueden agrupar en aquellos destinados al control del dolor y aquellos con efecto potencial para modificar el curso de la enfermedad (efecto estructural). Sin embargo; los medicamentos con efecto estructural, condromodulador o condroprotector único (denominados también DMOA por sus siglas en inglés: "Disease modifying OA drugs") como grupo es inexistente, porque ningún medicamento ha demostrado el potencial para remodelar o renovar la estructura de la articulación afectada por los cambios degenerativos propios de la osteoartritis [6].

Entre los medicamentos destinados al control del dolor, los de acción rápida alivian los síntomas y mejoran la capacidad funcional, pero no modifican la progresión de la enfermedad [7]. Entre este grupo de medicamentos, se encuentran aquellos que pueden administrarse por vía oral como el paracetamol, los antiinflamatorios no-esteroideos tradicionales (AINEs), los inhibidores selectivos COX-2 y los opioides, otros se pueden administrar por vía tópica (AINEs, capsaicina, etc.); y también por vía intraarticular (corticoides) [8].

Los medicamentos sintomáticos de acción lenta (denominados SYSADOAs por sus siglas en inglés: Symptomatic SlowActing Drugs for Osteoarthritis), como su nombre lo indica, tienen un inicio de acción más lento, pero también un efecto residual luego de suspenderlos [9]. Se reconoce que algunos de los SYSADOA podrían tener también efecto estructural [10]. Ambos tipos de medicamentos sintomáticos han demostrado eficacia, pero tienen propiedades y mecanismos de acción distinta (Tabla 1) [7].

Los SYSADOAs como grupo han despertado controversias notorias sobre su eficacia en el control de los síntomas; y sobre su posible efecto estructural; y por décadas han sido percibidos con desconfianza y escepticismo de parte de los

Tabla 1. Diferencias entre los medicamentos sintomáticos de acción rápida y lenta.

\section{Medicamentos sintomáticos de acción rápida}

Inicio de acción rápida

Efecto de rebote temprano al suspenderlos

Efecto temporal y dependiente de la dosis

No modifican el curso de la enfermedad ni la pérdida de cartílago articular

\section{Medicamentos sintomáticos de acción lenta (SYSADOAs)}

Inicio de acción lento

Eficacia persistente por algunas semanas luego de suspenderlos Permiten ahorrar dosis de analgésicos/AINEs

Existen evidencias que sugieren que podrían tener un potencial efecto modificador del curso de la enfermedad 
médicos. El propósito de la presente revisión es analizar las razones de esta controversia centrándonos en la eficacia sintomática de los SYSADOAs, no revisaremos ni discutiremos el potencial efecto modificador de la enfermedad de estos medicamentos.

\section{¿Cuáles son los objetivos terapéuticos que debe cumplir un SYSADOA?}

Dougados plantea que los SYSADOA deben cumplir 4 objetivos terapéuticos: Proporcionar alivio de los síntomas, disminuir el requerimiento de medicación sintomática concomitante (analgésicos, AINEs, etc.), prevenir el daño estructural y evitar (o retardar) el requerimiento de cirugía de reemplazo articular. Como se mencionó anteriormente el alivio de los síntomas característicamente tiene un inicio más lento con los SYSADOAs en comparación con los AINEs, pero su efecto residual se mantiene por un determinado periodo de tiempo después de suspenderlos (alrededor de 8 semanas) [11]. Disminuir el requerimiento de AINEs es uno de los beneficios más importantes que proporcionan los SYSADOAs, debido a que se reduce el riesgo de toxicidad por estos medicamentos [11, 12].

Prevenir la progresión del daño estructural implica de cierto modo modificar el curso de la enfermedad, pero Dougados comenta que para el clínico y los investigadores cumplir esta meta puede ser percibida de manera diferente, para el primero implica la disminución radiológica de la pérdida del espacio articular; mientras que los investigadores se focalizan más en demostrar la evidencia estadística del efecto estructural [11]. Adicionalmente valorar este objetivo ha estado limitado debido a la variabilidad de las diferentes técnicas destinadas a mesurar la progresión del daño estructural, lo cual ha dificultado interpretar el efecto de las diferentes intervenciones terapéuticas sobre la pérdida del cartílago articular [12]. Aún con estas discrepancias, algunos estudios clínicos sugieren que ciertos SYSADOAs podrían tener efecto estructural; sin embargo este aspecto hasta el momento es controversial [11, 13, 14, 15, 16].

Evitar o prevenir el requerimiento de cirugía de reemplazo articular es quizás el objetivo más importante para demostrar el efecto estructural de los SYSADOAs y su capacidad para modificar el curso de la enfermedad [11]; se encuentra evidencia disponible para el sulfato de glucosamina [17] y el sulfato de condroitina [18], aunque se considera que el tamaño de muestra necesario para alcanzar conclusiones significativas sobre este aspecto con la potencia suficiente, es considerablemente mayor a la empleada en algunos estudios clínicos [19].
Prevenir o retardar el requerimiento de la cirugía de reemplazo articular es un criterio muy atractivo debido a que se evitaría un número de procedimientos quirúrgicos con el subsecuente riesgo de complicaciones; así como la carga económica de los costos de la cirugía y la rehabilitación [20]. Sin embargo; el empleo de este parámetro se cuestiona también por la inconsistencia en decidir qué pacientes requieren cirugía, la falta de estandarización en las guías para indicar este procedimiento y la carencia de un adecuado número de estudios clínicos que evalúen este parámetro [20].

\section{Factores que influencian en la eficacia de los SYSADOAs}

La heterogeneidad observada en los desenlaces finales sobre la eficacia de los SYSADOA en los diferentes estudios clínicos ha sido materia de una amplia discusión; y en diferentes metanálisis, la variación encontrada en los resultados ha sido mayor que la esperada por el azar, de manera que conviene analizar las razones responsables de esta heterogeneidad [21].

Se reconoce que uno de los aspectos que ha impactado más en los resultados de eficacia es la baja calidad metodológica de muchos de los estudios clínicos disponibles, que podrían haber generado sesgos que dificultan alcanzar conclusiones y limitan el valor de extrapolar estos resultados a las guías de manejo y recomendaciones de consenso sobre los SYSADOAs [22]. Diferentes metanálisis de la base Cochrane y otros, demuestran una heterogeneidad considerable en los estudios clínicos con sulfato de glucosamina [21], sulfato de condroitina [23], insaponificables de aguacate y soja [24] y diacereína [25].

Adicionalmente a los factores relacionados al diseño del estudio, existen múltiples razones que pueden explicar al menos parcialmente los resultados variables de eficacia en los estudios con los SYSADOA, como las características y la naturaleza de la molécula específicamente empleada, las variaciones en la molécula original, el estatus de registro (medicamentos de venta libre o bajo prescripción), el procesamiento y la fuente de financiación de los estudios clínicos entre otros.

\section{Influencia de las características y naturaleza de la molécula}

Las sales de glucosamina han sido ampliamente empleadas en la osteoartritis; y existe una larga lista de estudios clínicos, en algunos de éstos se encuentra evidencia positiva de eficacia en el control de los síntomas [26, 27], mientras que otros la cuestionan $[28,29]$. 
Las sales más comúnmente empleadas son el sulfato de glucosamina y el clorhidrato de glucosamina, ambas moléculas difieren principalmente en la naturaleza de la sal incluida en su estructura para bloquear al protón del grupo amino de la glucosamina [30]. Se ha reconocido que se trata de dos moléculas con propiedades farmacocinéticas y farmacodinámicas distintas, a pesar de que ambas contienen glucosamina. Al comparar éstas presentaciones en modelos animales, Meulyzer encuentra que después de la administración oral a dosis equivalentes, el sulfato de glucosamina alcanza concentraciones significativamente mayores en el líquido sinovial en comparación con el clorhidrato de glucosamina, posiblemente por una mayor absorción y mejor biodisponibilidad, aunque aún permanece por determinar si esta diferencia se traduce en algún efecto biológico significativo [31].

Diferentes estudios clínicos, revisiones sistemáticas, metanálisis y opiniones de expertos coinciden en que el clorhidrato de glucosamina es ineficaz en controlar el dolor en la osteoartritis [32, 33, 34, 35]. Sobre este aspecto es importante comentar uno de los estudios clínicos más contradictorios con glucosamina. En el estudio GAIT, diseñado para valorar la eficacia de la glucosamina en la osteoartritis de rodillas, Clegg evaluó a 1,583 pacientes que fueron randomizados en cinco grupos, el primero recibió glucosamina (en forma de clorhidrato) a dosis de 500 mg 3 veces al día, el segundo recibió sulfato de condroitina a dosis de 400 mg 3 veces al día, el tercero recibió la combinación de ambos, el cuarto recibió celecoxib a dosis de $200 \mathrm{mg}$ al día y el último grupo recibió placebo. El objetivo primario de eficacia fue la reducción en el $20 \%$ en la subescala del dolor del WOMAC (The Western Ontario and McMaster Universities Osteoarthritis Index) desde el basal hasta la $24^{\circ}$ semana, e incluyó también otros parámetros secundarios de eficacia.

El objetivo primario se cumplió en el $60.1 \%$ en el grupo placebo, en el $64 \%$ en el grupo que recibió clorhidrato de glucosamina, en el $65.4 \%$ en el grupo que recibió sulfato de condroitina, en el $66.6 \%$ en quienes recibieron ambos productos; y en el $70.1 \%$ en el grupo que recibió celecoxib. Solo se encontró diferencia significativa en eficacia en el grupo que recibió celecoxib en comparación con placebo ( $P$ $=0.008$ ). Sin embargo; cuando se estratificó a los pacientes de acuerdo a la severidad de los síntomas, solo en aquellos con dolor moderado a severo que recibieron la combinación de glucosamina y condroitina se encontró una eficacia significativamente superior al placebo $(P=0.002)$. Los autores concluyeron que la glucosamina y la condroitina solas o administradas en conjunto no fueron eficaces en reducir el dolor en el grupo total de pacientes evaluados con osteoartritis de rodillas [29].
Los autores reconocieron que ciertos factores podrían haber influenciado en los resultados, como la alta tasa de respuesta con el placebo, 60\% en el estudio GAIT en comparación con el 30\% de lo esperado en estudios con otros medicamentos orales para la osteoartritis de rodillas [36]. Adicionalmente; el grado relativamente bajo del dolor en los participantes podría haber limitado la capacidad para detectar beneficios, o la falta de sensibilidad de los parámetros empleados no permitió detectar cambios o posibles diferencias en la eficacia entre los grupos [29]. Sin embargo; algunos autores consideran también que la pobre biodisponibilidad del clorhidrato de glucosamina podría explicar la falta de eficacia observada en el estudio GAIT [37, 38].

Los estudios clínicos con clorhidrato de glucosamina han mostrado un tamaño del efecto para el dolor pequeño o nulo y no significativo [21, 33]. Es más; la mayoría de estudios clínicos con desenlaces negativos de eficacia han empleado clorhidrato de glucosamina; y contrariamente aquellos con resultados positivos han empleado el sulfato de glucosamina a dosis de 1,500 mg [39]. Más aún; ninguna de la guías de manejo recomienda el clorhidrato del glucosamina, sino más bien el sulfato de glucosamina [40]. Es de esperarse por esta razón que un factor de confusión al analizar los resultados finales de eficacia con la glucosamina haya sido el tipo específico de molécula empleada al evaluar en conjunto los estudios con las sales de sulfato y clorhidrato.

\section{SYSADOAs: ¿Productos nutracéuticos de venta libre o medicamentos de prescripción?}

En algunos países los SYSADOAs son considerados productos nutracéuticos mientras que en otros, algunas de las presentaciones se encuentran registradas como medicamentos de prescripción, sin embargo no existe uniformidad entre las autoridades sanitarias de cada país sobre si considerar a los SYSADOAs como productos naturales, suplementos nutricionales o fármacos.

Es conveniente comentar que en el caso de los medicamentos, el proceso de registro se realiza de acuerdo a las regulaciones sanitarias establecidas para este propósito; mientras que en el caso de los suplementos nutricionales no se requiere cumplir las mismas condiciones [41].

Los medicamentos requieren un desarrollo a largo plazo (usualmente más de 10 años) con estudios pre-clínicos y clínicos; y su eficacia se evalúa en estudios clínicos apropiados, conducidos bajo los estándares de las Buenas Prácticas Clínicas [42]; mientras que el desarrollo de los suplementos nutricionales no es tan exigente. A diferencia de los productos naturales que se expenden sin requerimiento de prescripción 
médica, los medicamentos tienen un uso controlado por el médico y el farmacéutico; y un seguimiento estricto de seguridad, frente al uso no controlado de los productos naturales o con un seguimiento de seguridad variable de acuerdo a cada país.

La calidad de los nutracéuticos puede estar influenciada por la materia prima, las diferentes formas químicas, la sustitución inapropiada de materiales, la presencia de contaminantes, las diferentes formulaciones y la complejidad de los productos [43]. Como comenta Reginster, el mercado norteamericano está saturado de suplementos nutricionales de baja calidad, no manufacturados de acuerdo a los procedimientos farmacéuticos estándar, con un patrón farmacocinético pobre y un contenido variable del producto [27]

En Europa y algunos otros países, en los cuales se encuentran disponibles los productos originales (sulfato de glucosamina y sulfato de condroitina cristalizados), éstos son considerados de grado farmacéutico y aprobados por la EMEA (European Medicines Agency) o las autoridades sanitarias correspondientes; mientras que en otras regiones los únicos productos que contienen glucosamina o condroitina son considerados suplementos nutricionales [27]. Estos últimos carecen del mismo nivel de pureza que las formulaciones de prescripción, no son bioequivalentes con éstas y pueden no contener la concentración de la sustancia activa consignada en la etiqueta [42].

Este último es uno de los aspectos que remarca la pobre calidad de algunos suplementos nutricionales. Russell evaluó el contenido de 14 presentaciones de sulfato de glucosamina de venta libre; y encontró que el contenido variaba entre el $41 \%$ y $108 \%$ con respecto a lo consignado en el etiquetado [44]. Lockwood evaluó diferentes presentaciones comerciales de productos nutracéuticos y concluyó que la calidad de los mismos era baja; y encontró que en 5 de 12 presentaciones de la combinación de glucosamina y condroitina, en 2 de 4 presentaciones de glucosamina y en 3 de 3 presentaciones de condroitina, el contenido del producto estaba por debajo de lo consignado en la etiqueta [43]. Adebowale encontró una variación entre el contenido real y el consignado en la etiqueta de los productos que contenían glucosamina y condroitina disponibles en el mercado, con una variación que osciló entre el 0\% y el 115\%; y en aquellos con precios más bajos, encontró una seria deficiencia en alcanzar las concentraciones indicadas en la etiqueta (<10\% de lo esperado) [45].

Indudablemente, la diferencia en el estatus de dispensación del producto representa un sesgo importante cuando se analizan los resultados de eficacia al comparar estudios clínicos que incluyen preparados de venta libre, generando evidencia contradictoria y que contribuye a mantener posi- ciones discordantes con respecto a la eficacia y utilidad de los SYSADOAs. Se debe considerar también que no existe bioequivalencia entre los genéricos de glucosamina, de manera que los resultados con un preparado no pueden ser extrapolados a otros; y también las diferencias en la calidad de manufactura podrían explicar sus distintos efectos terapéuticos [46].

En base a la variabilidad en la eficacia demostrada con las diferentes formulaciones de las sales de glucosamina, el último consenso de la ESCEO recomienda la prescripción del sulfato de glucosamina cristalina patentada [15]; y el empleo de otras formulaciones diferentes se podría considerar hasta no ética; y desde el punto de vista socioeconómico un desgaste de recursos económicos [37]

Finalmente se debe tomar en cuenta que la mayoría de usuarios de productos naturales no los consideran como medicamentos y al no requerir una prescripción para adquirirlos, los perciben como seguros. Sin embargo; estos productos pueden asociarse a un riesgo de eventos adversos o interacciones medicamentosas, razón por la cual se debe preguntar de manera directa sobre el empleo de nutracéuticos durante los interrogatorios médicos [47]

\section{Influencia del fabricante y del procesamiento del producto}

En el caso de la glucosamina que es regulada como medicamento en Europa, el control de calidad de los productos de venta libre disponibles comercialmente ha sido una prerrogativa del fabricante [48]. La obtención de la glucosamina incluye varios pasos, pero como es de naturaleza hidroscópica e inestable, durante el proceso de extracción usualmente son cristalizadas en presencia de cantidades variables de cloruro de potasio o de sodio para mejorar la estabilidad, asimismo la cantidad de sal agregada no es constante y la dilución de los cristales de glucosamina durante el proceso de manufactura podría influenciar en la calidad del producto final [44, 48].

Es de esperarse que el proceso de manufactura de cada fabricante ejerza influencia en la naturaleza y pureza del producto terminado, que finalmente se podría traducir en la eficacia clínica, con las sales provenientes de diferentes casas farmacéuticas que emplean métodos de procesamiento distintos.

Towheed en un metanálisis Cochrane destinado a valorar la eficacia de la glucosamina en la osteoartritis, encontró que el preparado de una casa farmacéutica específica (Rottapharm) fue más eficaz que el placebo en reducir el dolor y mejorar la función en los pacientes con osteoartritis; mientras que al analizar los estudios con glucosamina provenientes de otros 
fabricantes, no encontró ninguna diferencia en eficacia sobre el placebo. El autor comentó que el sulfato de glucosamina que demostró eficacia estaba aprobado en Europa como medicamento de prescripción [49].

En el metanálisis de Eriksen también se encontró que la glucosamina proveniente de una casa farmacéutica (Rottapharm/ Madaus) fue superior en eficacia cuando se comparó con las provenientes de otros fabricantes. El autor atribuyó esta superioridad entre otras causas, a la naturaleza hidroscópica de la forma cristalina especialmente estabilizada producida por la casa farmacéutica mencionada [33].

Si el proceso de manufactura influencia en los productos que tienen un solo principio activo, es de esperarse que sea mayor en aquellos compuestos por múltiples moléculas en las cuales el principio activo final resulta de la cantidad y proporción de cada una de ellas, incluyendo las interacciones entre las mismas.

El insaponificable de aguacate y soja es considerado un producto medicinal derivado de fuentes vegetales que se obtiene después de un procedimiento de manufactura específico y patentado. El producto original está compuesto por una mezcla de fracciones insaponificables que son las sustancias aceitosas que permanecen después de la hidrólisis (saponificación), un tercio de las cuales la conforman aceites de aguacate y dos tercios aceites de soja. Los insaponificables de aguacate son extraídos del fruto de la Persea gratissima y los de soja de las semillas del Glycine max, gracias a un complejo proceso que se inicia con la selección de las frutas, desecación y extracción del aceite; y que finalmente resultan en el principio activo final denominado ASU Expanscience ${ }^{\mathrm{TM}}$. Las especificidades del producto y el proceso de obtención están protegidos por patentes [50].

A diferencia de los medicamentos de origen químico, los productos herbarios acabados contienen una mezcla compleja de diferentes compuestos con sustancias activas, que pueden ser sinérgicas, complementarias, antagonistas o tóxicas [51]. Esta aseveración implica que la acción terapéutica depende de la suma de todos los ingredientes que constituyen un preparado único y con un principio activo distinto en comparación con los productos individuales de los cuales deriva, lo cual confiere acciones terapéuticas y eventos adversos particulares para esa combinación [24].

Los beneficios de los productos herbarios acabados están influenciados por el proceso de manufactura, del cual depende la cantidad del principio activo en el producto final. Los cambios en la extracción del principio activo podrían resultar en cantidades reducidas y probablemente clínicamente inefectivas del principio activo [52]. También hay que considerar que las plantas que representan la fuente de estos produc- tos, pueden estar sometidas a cambios debidos a variables intrínsecas derivadas de la geografía, geología y el clima [53].

Estas razones hacen necesario conocer las diferentes características de cada producto herbario acabado, que incluya no solo las fuentes, los ingredientes y la composición final, sino además asegurarnos de que el proceso de manufactura haya sido el apropiado y estandarizado. Indudablemente en este contexto y considerando que el principio activo no solo es el resultado de la extracción de diferentes componentes herbarios, sino también del proceso de manufactura, no es conveniente extrapolar los resultados de estudios clínicos derivados de un producto herbario acabado a otros similares, pero que han empleado un proceso de manufactura diferente al descrito originalmente.

\section{Influencia de la fuente de financiación de los estudios clínicos con los SYSADOAS}

En los estudios clínicos que evalúan la eficacia de la glucosamina con la financiación o participación de la industria farmacéutica, usualmente se reportan resultados más positivos en eficacia y con un mayor tamaño del efecto en comparación con aquellos no financiados o ejecutados por investigadores independientes. Se ha considerado que la fuente de financiación es uno de los factores predictores más importantes de los resultados y también un sesgo potencial $[49,21]$.

El soporte de la industria en los estudios de investigación con productos farmacéuticos ha motivado un análisis extenso sobre el impacto de la financiación en los mismos. Se ha planteado que las conclusiones más positivas en los estudios financiados por organizaciones con fines de lucro o por la industria farmacéutica se deben a probables sesgos en la interpretación de los resultados; y algunos autores han encontrado una asociación estadísticamente significativa entre la financiación de la industria y las conclusiones en favor de los nuevos productos farmacéuticos [54, 55, 56].

Más aún; se ha reportado que en los estudios clínicos randomizados financiados por organizaciones de lucro, las conclusiones de los autores favorecen significativamente las intervenciones experimentales si existe un conflicto de interés declarado [57]; y tienden a recomendar el medicamento evaluado como el de elección [58]. Los factores que pueden explicar estos hallazgos radican en que en la mayoría de estudios financiados los productos activos se comparan contra placebo o contra ninguna intervención [57], o pueden radicar en la selección de un comparador inapropiado y en sesgos en la publicación [59].

Sin embargo; existe otra explicación tentativa para los resultados favorables en los estudios financiados por la in- 
dustria. Reginster comenta que las compañías farmacéuticas cuentan con profesionales con amplia experiencia en farmacología clínica, en confeccionar estudios con diseño apropiado, en evitar factores de confusión; y en satisfacer las demandas regulatorias. Sin embargo; quizás la razón más importante es que están obligados a seguir las normas de las Buenas Prácticas Clínicas y los estándares internacionales de investigación, con el monitoreo de los estudios clínicos y las auditorias correspondientes [35].

\section{Cuando iniciar el tratamiento con SYSADOAs}

La tendencia a emplear SYSADOAs parece ser variable entre las distintas regiones y también entre las diferentes especialidades. Prieto-Alhambra encontró que en España, al menos un tercio de los pacientes con diagnóstico reciente de osteoartritis inicia un SYSADOA, los usuarios nuevos generalmente son pacientes más jóvenes, con afección de rodillas y con un mejor perfil cardiovascular en comparación con aquellos que emplean paracetamol [60]; y en Francia los SYSADOAs son más prescritos por los reumatólogos que por los médicos generales (45.0\% vs. 39.1\%; $p<0.01$ ); mientras que éstos últimos tienden a prescribir dosis bajas de AINEs más frecuentemente que los reumatólogos [61].

En cambio en Alemania, Rosemann encontró que solo una pequeña proporción de pacientes con osteoartritis de rodillas empleaba SYSADOAs, generalmente de venta libre (alrededor del 10\%); y un porcentaje más bajo aún (alrededor del $3 \%)$ los recibía bajo prescripción médica [62]. En un hospital terciario en la India se reporta que los SYSADOAs fueron empleados solo en alrededor del $10 \%$ pacientes [63].

La posición de los SYSADOAs en el manejo de la osteoartritis es materia de discusión, pero es aún más difícil encontrar evidencia sobre el momento para iniciar estos productos en el paciente con osteoartritis. En el último consenso ESCEO, se recomiendan como el primer paso en el manejo conjuntamente con el paracetamol [15]. Esta posición es interesante por varias razones; en primer lugar los diferentes SYSADOAs han demostrado eficacia en reducir el consumo de AINEs; y en los adultos mayores este efecto se traduce en la reducción subsecuente del riesgo de eventos adversos asociados a su empleo [26, 27, 64, 65]. Adicionalmente, el empleo de los SYSADOAs no requiere de gastroprotección y es otra ventaja para este grupo poblacional [66].

Se podría especular que cuanto más tempranamente se inicien los SYSADOAs probablemente los beneficios sobre el cartílago articular podrían ser mejores, pero no hemos encontrado ninguna información científica disponible para afir- mar esta premisa. Sin embargo; solo el efecto analgésico y la capacidad de ahorrar AINEs, podrían ser razones suficientes para justificar el empleo temprano de los SYSADOAs en el curso de la enfermedad.

\section{Riesgo de eventos adversos asociados al empleo de los SYSADOAs}

El sulfato de glucosamina es bien tolerado y los eventos adversos usualmente son leves (nauseas, pirosis, diarrea, constipación, alergias dérmicas, cefalea). Un aspecto en controversia es el efecto de la glucosamina sobre el control de la glicemia y se recomienda monitorizar los niveles de glucosa plasmática en los diabéticos. Se debe tener precaución en los pacientes alérgicos a mariscos o crustáceos por las posibles reacciones alérgicas con la glucosamina, cuando el producto ha sido obtenido a partir de estas fuentes [67]. Dos estudios clínicos recientes reportaron que el sulfato de glucosamina elevó significativamente la presión intraocular especialmente en adultos mayores, los autores recomendaron tomar con precaución estos resultados y remarcaron la necesidad de estudios con mayor tamaño de muestra para evaluar esta asociación [68, 69].

Los eventos adversos asociados al empleo del sulfato de condroitina son escasos e incluyen reacciones alérgicas, constipación, náuseas, ardor epigástrico, diarrea, alopecia, edema de párpados o de miembros inferiores y extrasístoles. Se recomienda cuidado en los pacientes asmáticos y el uso intramuscular está contraindicado en sujetos con desórdenes de la coagulación o anticoagulados [67].

La diarrea relacionada al uso de la diacereína usualmente se presenta en las primeras dos semanas de tratamiento y empeora con el uso prolongado y el empleo de dosis diarias altas. Otros eventos adversos asociados al uso de la diacereína son los desórdenes de la piel como erupciones dérmicas y prurito. Se pueden presentar cambios en la coloración de la orina debido a la eliminación de metabolitos de la diacereína, sin embargo este último evento adverso no se considera clínicamente significativo [70]. Se ha reportado también elevación de las transaminasas y casos de hepatotoxicidad asociada al empleo de la diacereína [71, 70].

Debido al riesgo de diarrea severa, la EMEA ha sugerido limitar el empleo de la diacereína en personas mayores de 65 años, administrar inicialmente la mitad de la dosis $(50 \mathrm{mg} /$ día) y suspender el medicamento si se presenta diarrea. En pacientes con antecedentes de enfermedad hepática no se recomienda iniciar diacereína [71]. El Grupo de Trabajo de la ESCEO, después de revisar los estudios clínicos randomizados, 
considera que los beneficios de la diacereína sobrepasan los riesgos y debería considerarse como una opción entre los agentes disponibles para el tratamiento de la osteoartritis, con las medidas de precaución recomendadas [72].

Los eventos adversos más comunes asociados al empleo del insaponificable de aguacate y soja incluyen pirosis, nauseas, regurgitación, ardor epigástrico, dispepsia, constipación y diarrea; reacciones cutáneas como eczema, urticaria, fotosensibilidad y síndromes de hipersensibilidad. También se han reportado elevación de enzimas hepáticas, colitis y alteraciones plaquetarias, pero la mayoría de eventos adversos han sido de naturaleza transitoria [73 ]. Algunos pacientes pueden referir regurgitación con sabor a lípidos que puede evitarse al tomar la cápsula con alimentos.

A pesar de las controversias, los SYSADOAs son medicamentos que han demostrado eficacia en el control de los síntomas de la osteoartritis en estudios clínicos con adecuado diseño, aunque su efecto estructural aún no está completamente aceptado. Sin embargo a nuestro parecer y como mencionamos en líneas anteriores, el efecto sintomático y la capacidad de reducir el consumo de AINEs bastan para considerar a estos medicamentos en el arsenal terapéutico para esta enfermedad. Más aun, tomando en cuenta que el perfil de eventos adversos para los SYSADOAs es diferente al de los demás medicamentos con efecto sintomático.

\section{Referencias}

1. Abhishek A, Doherty M. Diagnosis and clinical presentation of osteoarthritis. Rheum Dis Clin N Am. 2013; 39: 45-66

2. Zhang W, Doherty M, Peat G, Bierma-Zeinstra SMA, Arden NK, et al. EULAR evidence based recommendations for the diagnosis of knee osteoarthritis. An Rheum Dis 2009; 69: 483-489

3. Sinusas K. Osteoarthritis: diagnosis and treatment. Am Fam Physician 2012, 85: 49-56

4. Hoogeboom TJ, Stukstette MJ, de Bie RA, Cornelissen J, den Broeder AA, et al. Non-pharmacological care for patients with generalized osteoarthritis: design of a randomized clinical trial. BMC Musculoskelet Disord 2010; 11: 142

5. Brosseau L, Rahman P, Toupin-April K, Poitras S, King J, et al. A systematic critical appraisal for non-pharmacological management of osteoarthritis using the appraisal of guidelines research and evaluation II instrument. PLOS ONE 2014; 9[1], e82986

6. Bobazc K. Pharmacologic treatment of hand-, knee and hiposteoarthritis. Wien Med Wochenschr 2013; 163: 236-242
7. Reginster JY, Avouac B, Gosset C. Clinical Evaluation of Drug Therapy. In; Reginster JY, PelletierJP. Martel-Pelletier, Henrotin Y, Crasborn L: Osteoarthritis. Clinical and Experimental Aspects. Berlin: Springer, Heidelberg. 1999. P. 421-430

8. Roubille C, Martel-Pelletier J, Pelletier JP. Osteoarthritis treatments: where do we stand at the moment? Medicographia 2013; 35: 172-180

9. Dougados M. Clinical assessment of osteoarthritis in clinical trials. Curr Opin Rheumatol 1995; 7: 87-91

10. Dougados M, Devogelaer JP, Annefeldt M, Avouac B, Bouvenot $G$. Recommendations for the registration of drugs used in the treatment of osteoarthritis. Ann Rheum Dis 1996; 55, 552-557

11. Dougados M. Symptomatic slow-acting drugs for osteoarthritis: what are the facts? Joint Bone Spine 2006; 73: 606-609

12. Abadie E, Ethgen D, Avouac B, Bouvenot G, Branco J, et al. Recommendations for the use of new methods to assess the efficacy of disease-modifying drugs in the treatment of osteoarthritis. Osteoarthritis Cartilage 2004; 12: 263-268

13. Reginster JY, Gillot V, Bruyere O, Henrotin Y. Evidence of nutriceutical effectiveness in the treatment of osteoarthritis. Curr Rheumatol Rep 2000; 2: 472-477

14. Maheu E, Cadet C, Marty M, Moyse D, Kerloch I, et al. Randomised, controlled trial of avocado-soybean unsaponifiable [Piascledine] effect on structure modification in hip osteoarthritis: the ERADIAS study. Ann Rheum Dis. 2014; 73: 376-384

15. Bruyère $O$, Cooper $C$, Pelletier JP, Maheu E, Rannou F. A consensus statement on the European Society for Clinical and Economic Aspects of Osteoporosis and Osteoarthritis [ESCEO] algorithm for the management of knee osteoarthritis - from evidence-based medicine to the real-life setting. Semin Arhtritis Rheum 2016; 45: S3-S11

16. Rillo $O$, Riera $H$, Acosta $C$, Liendo $V$, Bolaños J, et al. PANLAR consensus recommendations for the management in osteoarthritis of hand, hip, and knee. J Clin Rheumatol 2016; 22: 345-354

17. Bruyère $O$, Pavelka K, Rovati LC, Gatterova J, Giacovelli G, et al. Total joint replacement after glucosamine sulphate treatment in knee osteoarthritis: results of a mean 8-year observation of patients from two previous 3-year, randomised, placebocontrolled trials. Osteoarthritis Cartilage 2008, 16: 254-260

18. Raynauld JP, Martel-Pelletier J, Dorais M, Haraoui B, Choquette D, et al. [2013]. Total knee replacement as a knee osteoarthritis outcome: predictors derived from a 4-year long-term observation following a randomized clinical trial using chondroitin sulfate. Cartilage, 4[3], 219-226

19. Ried JS, Flechsenhar K, Bartnik E, Crowther D, Dietrich A, et al. Sample size calculations for detecting disease-modifying osteoarthritis drug effects on knee replacement incidence in clinical trials: Data from the osteoarthritis initiative. Arthritis Rheumatol 2015; 67: 3174-3183

20. Altman RD, Abadie E, Avouac B, Bouvenot G, Branco J. Total joint replacement of hip or knee as an outcome measure for structure modifying trials in osteoarthritis. Osteoarthritis Cartilage 2005; 13: $13-19$ 
21. Vlad SC, LaValley MP, McAlindon TE, Felson DT. Glucosamine for pain in osteoarthritis: Why do trial results differ?. Arthritis Rheum 2007; 56: 2267-2277

22. Vasiliadis HS, Tsikopoulos K. Glucosamine and chondroitin for the treatment of osteoarthritis. World J Orthop 2017; 8: 1-11

23. Singh JA, Noorbaloochi S, MacDonald R, Maxwell LJ. Chondroitin for osteoarthritis. The Cochrane database of systematic reviews. 2015; 1:CD005614. doi:10.1002/14651858.CD005614.pub2.

24. Cameron M, Chrubasik S. Oral herbal therapies for treating osteoarthritis. Cochrane Database Syst Rev. 2014; 5: CD002947. doi:10.1002/14651858.CD002947.pub2

25. Fidelix TS, Macedo CR, Maxwell LJ, Fernandes Moça Trevisani V. Diacerein for osteoarthritis. Cochrane Database of Systematic Reviews 2014, Issue 2. Art. No.: CD005117. DOI: 10.1002/14651858.CD005117.pub3.

26. Towheed TE, Maxwell L, Anastassiades TP, et al. Glucosamine therapy for treating osteoarthritis. Cochrane Database Syst Rev 2009;2:CD002946

27. Reginster JY, Cooper C, Hochberg M, Pelletier JP, Rizzoli R, et al. Comments on the discordant recommendations for the use of symptomatic slow-acting drugs in knee osteoarthritis. Curr Med Res Opin 2015; 31: 1041-1045

28. McAlindon T, Formica $M$, LaValley $M$, Lehmer $M$; Kabbara K. Effectiveness of glucosamine for symptoms of knee osteoarthritis: results from an internet-based randomized double-blind controlled trial. Am J Med 2004; 117: 643-649

29. Clegg DO, Reda DJ, Harris $C L$, Klein MA, O'Dell JR, et al. Glucosamine, chondroitin sulphate and the two in combination for painful knee osteoarthritis. N Engl J Med 2006; 354: 795808

30. Block JA, Oegema TR, Sandy JD, Plaas A. The effects of oral glucosamine on joint health: is a change in research approach needed? Osteoarthritis Cartilage 2010; 18: 5-11

31. Meulyzer $M$, Vachon $P$, Beaudry $F$, Vinardell $T$, Richard $H$, et al. Comparison of pharmacokinetics of glucosamine and synovial fluid levels following administration of glucosamine sulphate or glucosamine hydrochloride. Osteoarthritis Cartilage 2008; 16: 973-979

32. Wu D, Huang Y, Gu Y, Fan W. Efficacies of different preparations of glucosamine for the treatment of osteoarthritis: a metaanalysis of randomised, double-blind, placebo-controlled trials. Int J Clin Pract 2013; 67: 585-594

33. Eriksen $P$, Bartels EM, Altman RD, Bliddal $H$, Juhl $C$, et al. Risk of bias and brand explain the observed inconsistency in trials on glucosamine for symptomatic relief of osteoarthritis: a metaanalysis of placebo-controlled trials. Arthritis Care Res 2014; 66: 1844-1855

34. Saengnipanthkul S, Waikakul S, Rojanasthien S, Totemchokchyakarn K, Srinkapaibulaya A, et al. Differentiation of patented crystalline glucosamine sulfate from other glucosamine preparations will optimize osteoarthritis treatment. Int J Rheum Dis 2017. doi:10.1111/1756-185X.13068
35. Reginster JY. The efficacy of glucosamine sulfate in osteoarthritis: financial and nonfinancial conflict of interest. Arthritis Rheum 2007; 56: 2105-2110

36. Reginster JY, Neuprez A, Lecart MP, Sarlet N, Bruyère $O$. Role of glucosamine in the treatment for osteoarthritis. Rheumatol Int 2012; 32: 2959-2967

37. Kucharz EJ, Kovalenko V, Szántó S, et al. A review of glucosamine for knee osteoarthritis: why patented crystalline glucosamine sulfate should be differentiated from other glucosamines to maximize clinical outcomes. Curr Med Res Opin 2016; 32: 9971004

38. Persiani S, Rotini R, Trisolino G, Rovatti LC, Locatelli M, et al. Synovial and plasma glucosamine concentrations in osteoarthritic patients following oral crystalline glucosamine sulphate at therapeutic dose. Osteoarthritis Cartilage 2007; 15: 764-72

39. Bruyère $O$, Cooper C, Al-Daghri NM, Dennison EM, Rizzoli R, et al. Inappropriate claims from non-equivalent medications in osteoarthritis: a position paper endorsed by the European Society for Clinical and Economic Aspects of Osteoporosis, Osteoarthritis and Musculoskeletal Diseases [ESCEO]. Aging Clin Exp Res. 2017. doi: 10.1007/s40520-017-0861-1

40. Henrotin $Y$, Lambert $C$. Chondroitin and glucosamine in the management of osteoarthritis: An update. Curr Rheumatol Rep 2013; 15: 361. Doi 10.1007/s11926-013-0361-z

41. Sáenz-Campos D. Medicamentos, plantas medicinales y productos naturales. Fármacos 2003; 16: 13-20

42. Cutolo M, Berenbaum F, Hochberg M, Punzi L, Reginster JY. Commentary on recent therapeutic guidelines for osteoarthritis. Semin Arhtritis Rheum 2015; 44: 611-617

43. Lockwood GB. The quality of commercially available nutraceutical supplements and food sources. J Pharm Pharmacol 2011; 63: 3-10

44. Russell AS, Aghazadeh-Habashi A, Jamali F. Active ingredient consistency of commercially available glucosamine sulfate products. J Rheumatol 2002; 29: 2407- 2409

45. Adebowale AO, Cox DS, Liang Z, Eddington ND. Analysis of glucosamine and chondroitin sulfate content in marketed products and the caco-2 permeability of chondroitin sulfate raw materials. JANA 2000; 3: 37-44

46. Gregory PJ. The recommendations for glucosamine do not tell the whole story: comment on the article by Hochberg et al. Arthritis Care Res 2013; 65: 326-327

47. Franco S, Gonzáles P. Los suplementos dietéticos y el anestesiólogo: resultados de investigación y estado del arte. Rev Colomb Anestesiol 2014; 42: 90-99

48. Aghazadeh-Habashi A, Jamali, F. The glucosamine controversy; a pharmacokinetic issue. J Pharm Pharmaceut Sci 2011; 2: 264273

49. Towheed T, Maxwell L, Anastassiades TP, Shea B, Houpt JB, et al. Glucosamine therapy for treating osteoarthritis. Cochrane Database of Systematic Reviews 2005, Issue 2. Art. No: CD002946. DOI:10.1002/14651858.CD002946.pub2. 
50. Msika P, Baudouin C, Saunois A, Bauer T. Avocado/soybean unsaponifiables, ASU EXPANSCIENCE, are strictly different from the nutraceutical products claiming ASU appellation. Osteoarthritis Cartilage 2008; 16: 1275-1276

51. Loew D, Kaszkin M. Approaching the problem of bioequivalence of herbal medicinal products. Phytother Res 2002; 16: 705-711

52. Cameron M, Gagnier JJ, Little CV, Parsons TJ, Blümle $A$, et al. Evidence of effectiveness of herbal medicinal products in the treatment of arthritis. Part 1: Osteoarthritis. Phytother Res 2009; 23: 1497-1515

53. Chrubasik S, Conradt C, Black A. The quality of clinical trials with Harpagophytum procumbens. Phytomedicine 2003; 10: 613-623

54. Bekelman JE, Li Y, Gross CP. Scope and impact of financial conflicts of interest in biomedical research: a systematic review. JAMA 2003; 289: 454-465

55. Bhandari M, Busse JW, Jackowski D, Montori VM, Schunemann $\mathrm{H}$, et al. Association between industry funding and statistically significant pro-industry findings in medical and surgical randomized trials. CMAJ 2004; 170: 477-480

56. Ahn R, Woodbridge A, Abraham A, Saba S, Korenstein D, et al. Financial ties of principal investigators and randomized controlled trial outcomes: cross sectional study. BMJ 2017; 356: i6770. doi: 10.1136/bmj.i6770

57. Kjaergard LL, Als-Nielsen B. Association between competing interests and authors' conclusions: epidemiological study of randomized clinical trials published in the BMJ. BMJ 2002; 325: 249-252

58. Als-Nielsen B, Chen W, Gluud C, Kjaergard LL. Association of funding and conclusions in randomized drug trials: a reflection of treatment effect or adverse events? JAMA 2003; 290: 921928

59. Lexchin J, Bero LA, Djulbegovic B, Clark O. Pharmaceutical industry sponsorship and research outcome and quality: systematic review. BMJ 2003; 326: 1167-1170

60. Prieto-Alhambra D, Herrero M, Vergés J. Trends of use of different SYSADOA in patients with osteoarthritis: a populationbased cohort study (abst) Osteoarthritis Cartilage 2013; 21, S164-S165

61. Richette $P$, Hilliquin $P$, Bertin $P$, Carni $P$, Berger $V$, et al [2011]. Comparison of general practitioners and rheumatologists' prescription patterns for patients with knee osteoarthritis. BMC Musculoskelet Disord 2011; 12: 72

62. Rosemann T, Laux G, Szecsenyi J. Osteoarthritis: quality of life, comorbidities, medication and health service utilization assessed in a large sample of primary care patients. J Orthop Surg Res 2007; 2: 12

63. Ullal SD, Sanji N, Kamath RK, Pai MRSM, Kamath SU, et al. Prescribing pattern for osteoarthritis in a tertiary care hospital. J Clin Diagn Res 2010; 4: 2421-2426
64. Appelboom T, Schuermans J, Verbruggen G, Henrotin $Y$, Reginster JY. Symptoms modifying effect of avocado/soybean unsaponifiables [ASU] in knee osteoarthritis. A double blind, prospective, placebo-controlled study. Scand J Rheumatol 2001; 30: 242-247

65. Henrotin Y, Marty M, Mobasheri A. What is the current status of chondroitin sulfate and glucosamine for the treatment of knee osteoarthritis? Maturitas 2014; 78: 184-187

66. du Souich P. Absorption, distribution and mechanism of action of SYSADOAS. Pharmacol Ther 2014; 142: 362-374

67. Glucosamine Sulfate [Herbs/Suppl] drug monograph. Medscape Drugs \& Diseases. Published 2017. Accedido el 28 de diciembre del 2017 Available at: https://reference.medscape.com/ drug/2-aminodeoxyglucose-sulfate-amino-monosaccharideglucosamine-sulfate-344469.

68. Murphy RK, Ketzler L, Rice RD, Johnson SM, Doss MS, et al. Oral glucosamine supplements as a possible ocular hypertensive agent. JAMA Ophthalmol. $2013 ; 131]$ : 955-957

69. Esfandiari H, Pakravan M, Zakeri Z, Ziaie S, Pakravan P, et al. Effect of glucosamine on intraocular pressure: a randomized clinical trial. Eye [Lond]. 2017; 31: 389-394

70. Panova $E$, Jones $G$. Benefit-risk assessment of diacerein in the treatment of osteoarthritis. Drug Saf 2015; 38: 245252.

71. European Medicines Agency [EMEA]. 2014. Restrictions to the use of diacerein-containing medicines. Restrictions intended to limit risks of severe diarrhoea and effects on the liver. 4 September 2014. EMA/544268/2014. Accedido el 28 de diciembre del 2017. http://www.ema.europa.eu/docs/en_GB/ document library/Referrals document/Diacerein/European Commission final decision/WC500173144.pdf

72. Pavelka K, Bruyère $O$, Cooper $C$, Kanis JA, Leeb BF, et al. Diacerein: benefits, risks and place in the management of osteoarthritis. An opinion-based report from the ESCEO. Drugs Aging 2016; 33: 75-85

73. Christiansen BA, Bhatti S, Goudarzi R, Emami S. Management of Osteoarthritis with Avocado/Soybean Unsaponifiables. Cartilage 2015; 6:30-44

\section{Publique en Acta Reumatológica}

Acta Reumatológica es una revista que tiene por fin la difusión de estudios clínicos relacionados con aspectos prácticos del diagnóstico, tratamiento y seguimiento de pacientes con patología reumatológica, de estudios epidemiológicos relacionados con patología inflamatoria y musculoesquelética de presentación común o infrecuente en la práctica clínica tanto en población adulta como pediátrica, de casos clínicos de patología poco habitual o de presentaciones inhabituales de patología frecuente, de imágenes didácticas e ilustrativas en reumatología y del estado actual e innovación en la formación especializada en reumatología. 